\title{
Elettronika Virtual Network Management
}

\author{
doi:10.3991/ijoe.v5i2.902
}

\author{
Alexandra Teodor ${ }^{1}$, Attila Valadi ${ }^{1}$, Doru Ursutiu ${ }^{2}$, Giuseppe Modugnio ${ }^{3}$, Marco Fiore ${ }^{3}$ \\ ${ }^{1}$ Elettronika Research S.R.L., Brasov, Romania, ${ }^{2}$ Transilvania University, Brasov, Romania \\ 3 Elettronika S.R.L., Palo del Colle, Italy
}

\begin{abstract}
This article introduces a simple and efficient implementation of a remote control system for the telecommunication networks. The paper deals with the automatization of transmission sites, the unattended monitoring and the ease of management of those. For the hardware's remote control we introduce a user friendly software interface between the user and the stations.
\end{abstract}

\section{Index Terms-RCU, ERCoS, GenIO, RCS.}

\section{INTRODUCTION}

For the management of the network, Elettronika has produced a remote control system that consists of two fundamental parts: the unit by Elettronika called RCU (Remote Control Unit), to be installed at the remote station and the ERCoS software to be installed on the computer at the control centre.

\section{REMOTE CONTROL UNIT}

\section{A. Description}

The remote Control Unit (RCU) is the hardware that must be placed at a station in order to control it at distance. It is designed to control remotely equipments made by Elettronika S.R.L.

Using WINDOWS OS interface you can control and monitor basic functions of the equipment. You have the possibility to read the current status of the equipments and the status history and change the operating parameters.

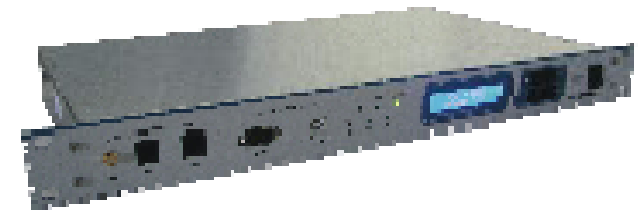

Figure 1. Remote Control Unit

\section{B. Main features}

There are available three models depending on the type of connection to the control center. Only one type of connection is available in every model, besides the standard PC interfaces (RS232/USB).

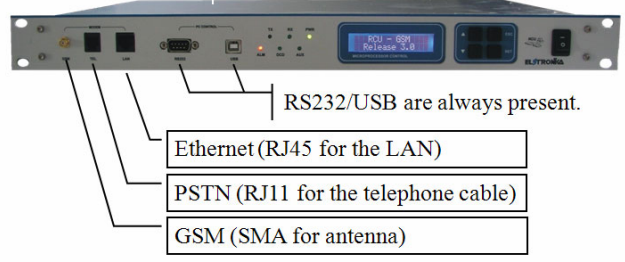

Figure 2. RCU-Connection types
The block diagram of the Remote Control Unit is shown in the figure below:

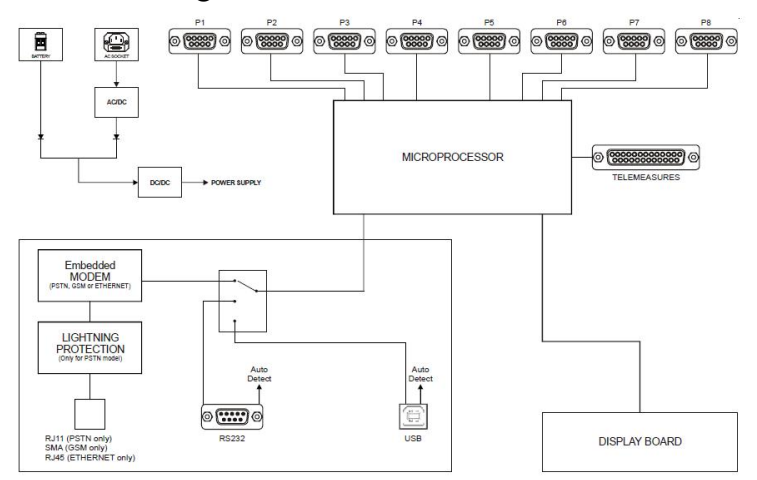

Figure 3. Block Diagram

\section{Firmware upgrade}

The microcontroller firmware of the RCU can be upgraded to later versions without removing the equipment from the rack or replacing any chip. For this you only need a Windows PC with a RS232 port and a serial null modem cable. Use direct RS232 connection to PC.

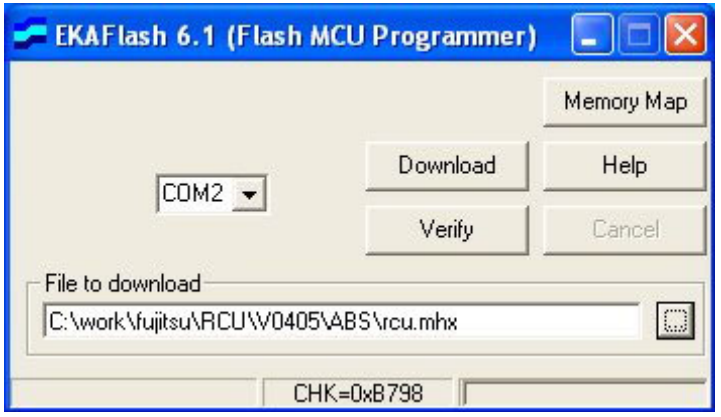

Figure 4. Firmware upgrade

\section{Telemeasure feauture}

The RCU controls up to 8 devices with RS485 connection, made by Elettronika. Because there are available 4 digital inputs, 4 digital outputs and 3 analog inputs as well, the unit can also perform telemeasure applications.

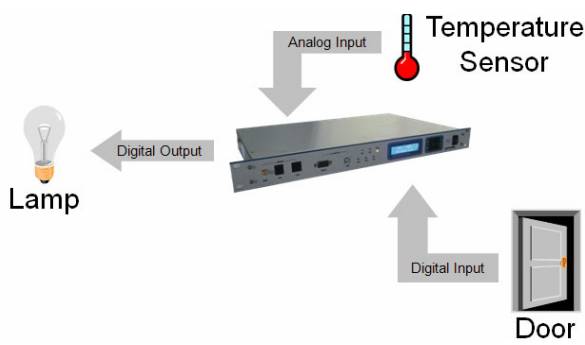

Figure 5. RCU-Telemeasures 


\section{ERCOS}

\section{A. Introduction}

ERCoS (Elettronika Remote Control Software) is the remote control software for the management of all Elettronika apparatuses through a single interface.

ERCoS allows connecting to a remote station directly by means of a serial cable, through a modem or Ethernet. While connected, the user can monitor the status of each individual device inside the station, and even change its parameters (on/off status, broadcasting power, frequency, etc).

When enabled, ERCoS monitors incoming calls on a telephone line, dedicated or not, or Ethernet coming from a remote RCU communicating some event (alarm or status change). This way the user does not have to connect continuously to the remote station in order to check that the equipment is working correctly.

The strength of ERCoS lies in its user-friendly graphical interface allowing an immediate understanding of its functions and an easy reading of all the remotelycontrolled data. Besides, the user may customize his network of Elettronika stations within ERCoS and use a single application to manage all of them. The automatic answer to alarm calls (even on dedicated lines) and the autopolling of the remote stations, make ERCoS even more useful and effective.

\section{B. Main Window}

The main window of ERCoS is composed by two elements which are generally referred to as Structure (left area) and View (right area).

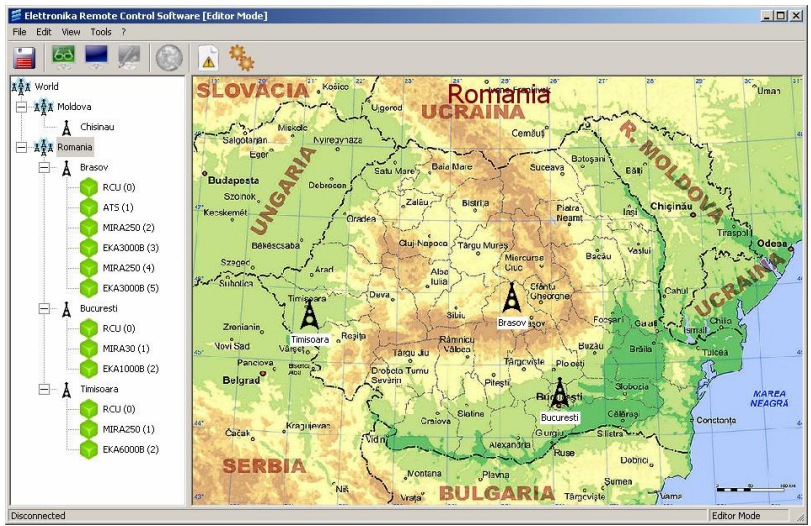

Figure 6. ERCoS Main Window

To better organize displayed information, especially when a large number of remote stations is used, it is possible to group sites, for instance grouping them by territory (maps or geographical regions) or depending on the broadcasting station, or according to any other rule. There are two grouping levels in ERCoS, called main map and secondary maps (or simply maps or regions). The main map contains several secondary maps, which in turn contain the stations.

This creates a tree structure with the root represented by the main map, secondary maps as second level nodes and within them the remote stations that are the third level nodes. Finally, the stations are composed by Elettronika equipments which are the fourth level nodes of the Structure. The picture below shows an example of a network.

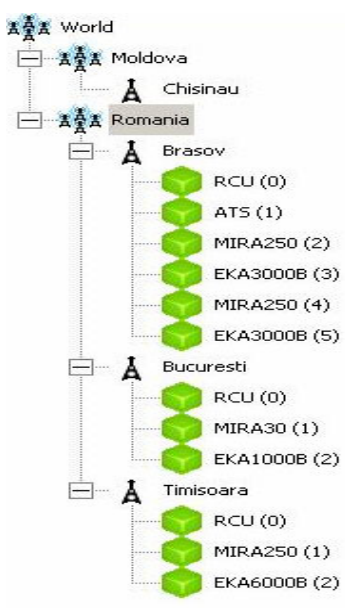

Figure 7. ERCoS Structure

The main map, called "World", contains all of the remote stations, grouped within two secondary maps.

The region called "Romania" contains three stations: "Brasov", a 3000W FM Elettronika station, provided with an antenna changeover with a redundant transmitter and amplifier, "Bucuresti", a 1000W FM station and "Timisoara" with a $6000 \mathrm{~W}$ FM station.

The sub-map "Moldova" only contains an empty station called "Chisinau".

Each station is composed by a variable number of devices by Elettronika (transmitters, amplifiers and so on). Before connecting to the remote station, its composition has to be defined by adding the various devices it contains. In order to add an apparatus to the station, select New Device from the popup menu shown above and choose your equipment from the window that appears.

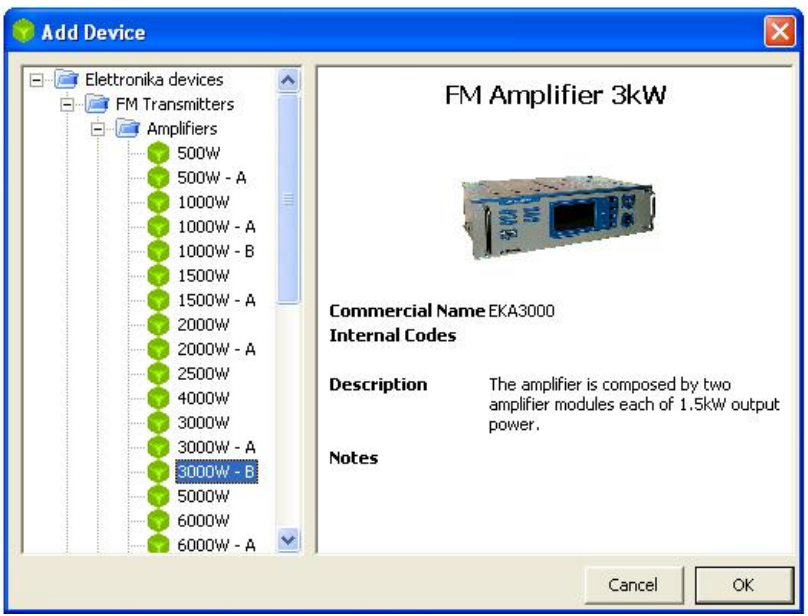

Figure 8. Adding new devices

Selecting for instance the "Brasov" station in the Tree Structure, the View area shows the composition of the station, as in figure 9.

By pressing F2 key, it is possible to change the device name. The number in parentheses is the port of the RCU to which the device is connected. This can be changed by right-clicking on the icon of the device and choosing Change Port Device from the context menu, as shown in figure 10 . 


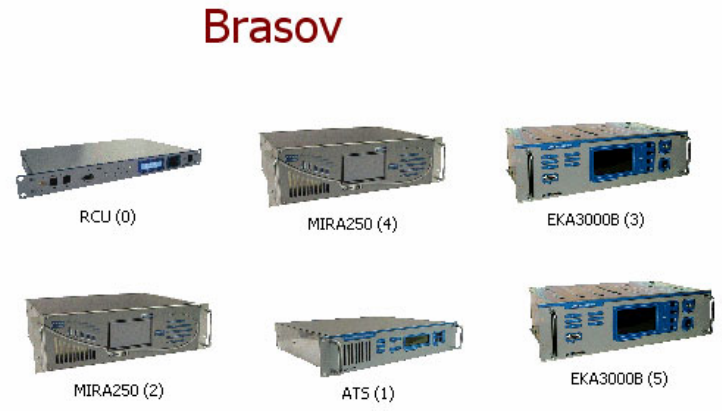

Figure 9. Devices contained in Brasov

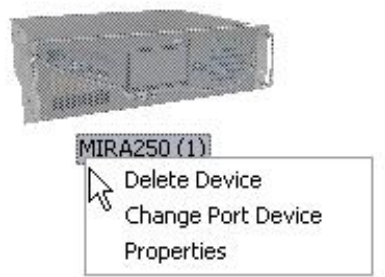

Figure 10. Change device properties

The same context menu also allows to remove a device from the station by choosing Delete Device, or to change its properties by choosing Properties. The object properties may also be modified double-clicking on the relevant icon. The properties window changes depending on the selected apparatus.

In some cases the user doesn't know or doesn't remember the composition of the site: which device is connected to which RCU port. Starting from ERCoS 3.0.1 a new feature is added to help the user compose its station, even from remote.

The AutoDetect is a new feature introduced with ERCoS 3.0.1 that can help the user to configure the station without knowing or remembering which Elettronika equipment is connected to which RCU port. The auto detecting process is possible only if a RCU is present in the site, so the first thing to do is to create a new site and add a RCU object. Also the communication settings must be set so ERCoS con make a connection with the RCU.

In order to perform Auto Detecting, switch to View or Monitor Mode and make a connection to the RCU. After the connection is made, click on the button on the toolbar to start AutoDetect (Editor Mode password must be typed in). During this operation, ERCoS tries to recognize the device connected to each RCU port and shows progressively the results in a pop-up window (see figure 11). For each port, the following can be written:

- None: no device is connected;

- Unknown: a device is connected, but ERCoS can't recognize it;

- Name of the device recognized.

All of ERCoS functions may be selected from the menu bar and some operations may be also easily made by using the toolbar, as show in figure 12.

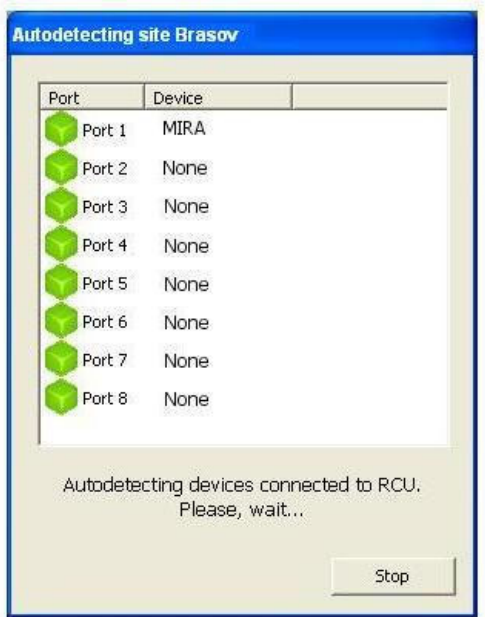

Figure 11. Devices contained in Brasov

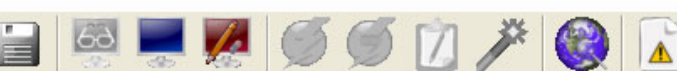

Figure 12. Toolbar

\section{Usage}

ERCoS is a generic software to control remote stations equipped with devices manufactured by Elettronika. Before connecting to a station, the user has to configure his network of Elettronika equipments, creating the stations and setting their composition. ERCoS provides three working modes to do this: View Mode, Monitor Mode and Editor Mode.

In Editor Mode it is possible to create the network of the remote stations to be controlled. Then it is possible to create and configure the maps, the sites and the devices within them.

View Mode and Monitor Mode are similar to each other and are normally used to connect to remote stations. While connected to a station, in View Mode you can only check the status and settings of the apparatuses, while in Monitor Mode you can also change status (such as switching the apparatus on or off).

During communication, ERCoS query all the equipments in the site, one after the other. In certain situation, one or more equipments can be off (for example, in dual-drive transmitters) and the communication with these failed after a timeout period. In order to avoid this, right-click on the equipment switched-off and select "Skip" item from context menu. From now on, that equipment will be skipped by ERCoS and the communication will not fail. Select again "Skip" item from the same menu to query again the equipment. The most important parameters of each device are displayed next to it: for example, working frequency and forward power for the FM transmitter MIRA, forward and reflected power for the amplifier EKA6000. Lack of communication between RCU and device is indicated by a "No Comm." label. The detailed parameters of any individual device may be seen by double-clicking the relevant icon: for example, double-clicking the icon of the EKA6000B will bring forth the following window. 


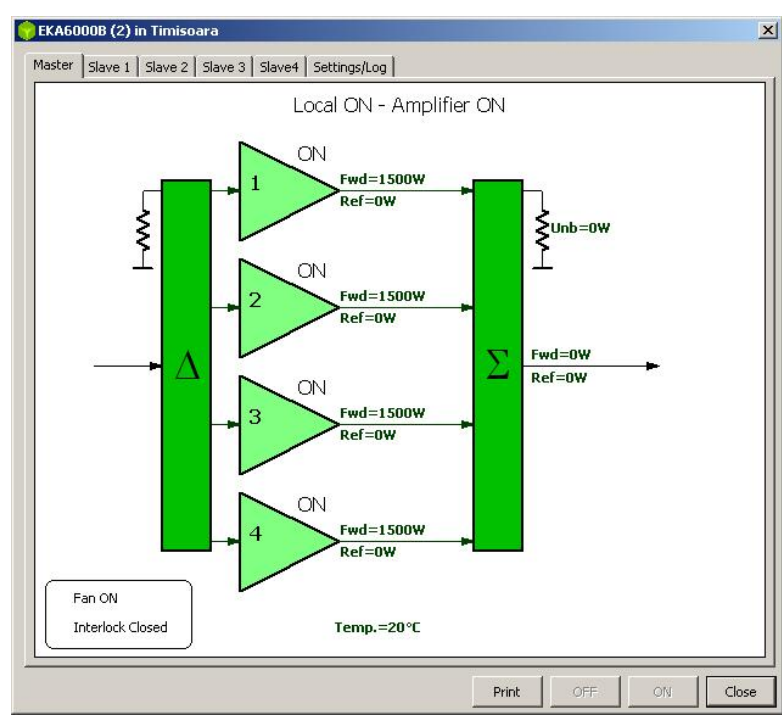

Figure 13. Internal Structure of the EKA 3000B device

This window allows monitoring the ON/OFF status of the whole amplifier (currently ON) and the status of the main switch of the amplifier (currently ON). Since the EKA6000B is composed by four $1500 \mathrm{~W}$ amplifiers, it is possible to control forward and reflected power for any individual block, as well as the global output power of the amplifier. It is possible to monitor an individual amplifier block in detail by either clicking on it or choosing the relevant tab. The window below is the detail of block 1:

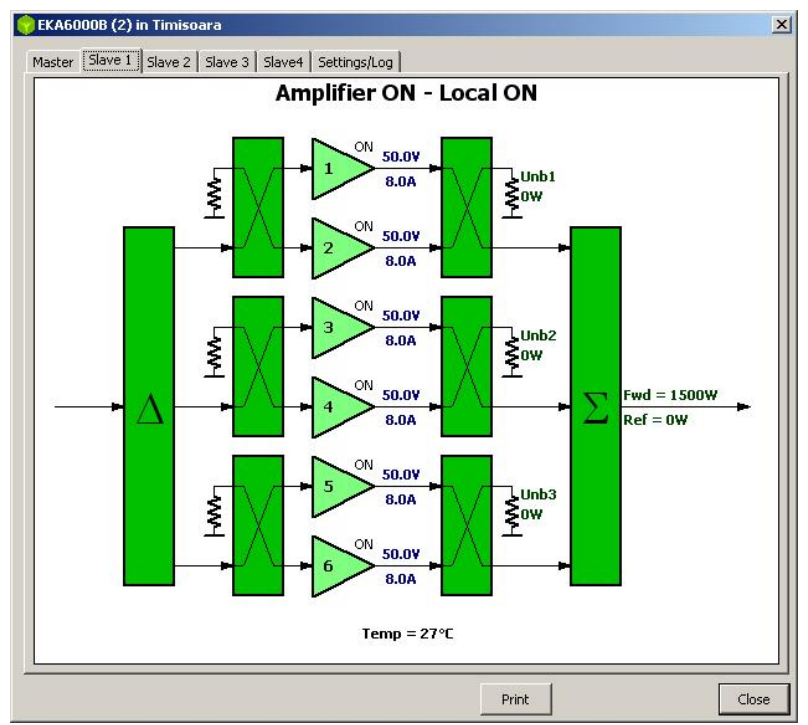

Figure 14. Internal Structure of the EKA 3000B slave 1

It is possible to check the value of each parameter and the relevant alarm status, if any (red LED lit next to the parameters).

For the EKA6000B it is possible to act remotely by means of the On and Off buttons, which allow to switch the amplifier on and off (both buttons are not enabled in View Mode). Besides, it is possible to read the history of the alarms stored into the control module, by selecting the Alarms History tab. It is possible to copy the list of events in the clipboard by selecting the items and selecting Copy menu that appears right clicking on the list.

While connected to a remote station, it is possible to generate a status report at any moment by clicking on the $\square$ button in the tool bar of the main window. ERCoS will automatically create the report including all parameters of currently connected devices, and will display it in a new window.

ERCoS starts in View Mode. To enter Monitor Mode or Editor Mode a customizable password must be typed this in order to prevent unauthorized people from changing the settings of the software or connecting to the transmitters and change their status. If the computer of the control centre has to be left uncontrolled, it is better to leave ERCoS in View Mode. It is possible to know the current mode at any moment by looking at the title bar or the status bar of the window.

\section{AutoAnswer}

The remote control unit ( $\mathrm{RCU}$ ) can be configured to automatically connect to the control centre to inform it about occurring alarms (lack of communication with some devices or signals outside the normal intervals) or other events (change of some input signals). To use this feature, the RCU must be correctly configured, enabling the alarm calls. The ERCoS must as well be configured to continuously monitor incoming connections.

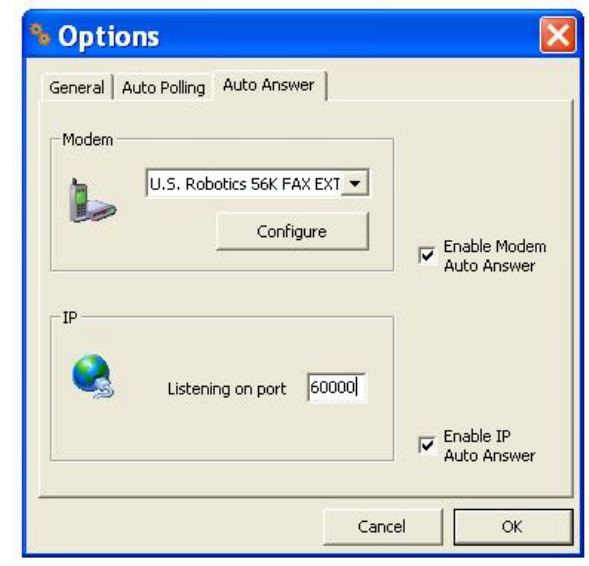

Figure 15. AutoAnswer window

The graphical interface allows enabling the reception of incoming connections on a specific modem to be selected and configured or on TCP/IP.

ERCoS is able to use two separate devices (thus two separate telephone lines) for incoming and outgoing calls to and from the control centre. This means that it is possible to connect two modems and two telephone lines to the same PC and use one of them to connect to remote station while the other to receive incoming alarm calls. This way when a station tries to inform the control centre of an alarm it will never find the line busy.

If AutoAnswer is enabled on TCP/IP, the listening TCP port must be specified. ERCoS will listen on this port for incoming connections from remote RCU (Ethernet model). Of course, the chosen TCP port must be free (no other software should use it) and unblocked by any firewall. This listening port for AutoAnswer must coincide with the TCP output port configured in the RCU-Ethernet.

\section{E. AutoPolling}

Another interesting feature made available to the user is the so called Autopolling. An alarmed station may indeed communicate with the control centre (that is with ERCoS) only if the remote control unit is working. In case it is the 
RCU itself to be in alarm for a failure, it would not be able to inform the control centre of the problem and the user may not notice the situation until he tries to connect to the station manually.

In order to be aware of this kind of alarms, the user should perform a manual polling of each and all remote stations in order to check that they are working properly. ERCoS provides the user with the Autopolling feature which automatically polls all the remote stations every day at set times.

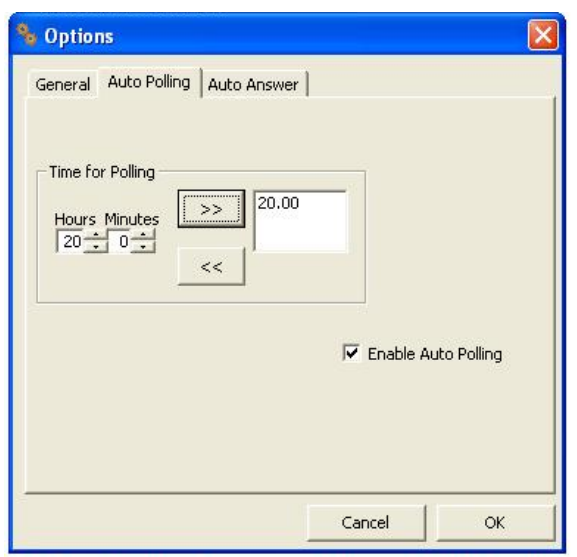

Figure 16. AutoPolling window

\section{COMPATIBILITY WITH OTHER VENDORS}

All though Elettronika recommends using only their own equipments it is also possible to interconnect devices manufactured by other companies. This can be done using the GenIO (General Input/Output) gateway specially developed for the needs of our customers. GenIO expands RCU telemeasures up to 4 digital inputs, 4 digital outputs, 4 analog inputs.

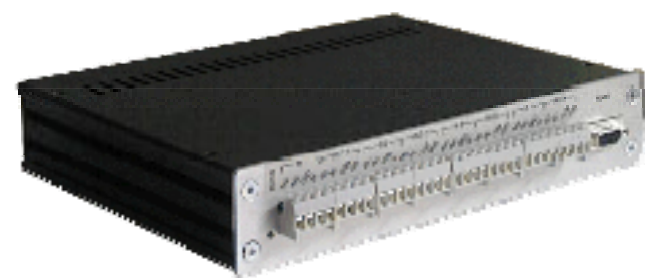

Figure 17. GenIO

\section{CONCLUSIONS}

The Remote Control System (RCS), one of Elettronika leading products, has been having an increasing success since its first appearance on the market.
The RCS allows controlling the status of all sites of a broadcasting network, trough a connection established by means of a telephone or GSM modem. The RCS system is composed by two elements, the RCU (Remote Control Unit) and ERCoS (Elettronika Remote Control Software).

The former is the hardware to be installed at each site, which allows to control up to 8 Elettronika devices. It automatically monitors the status of the connected devices and informs the control centre in case of alarm, even by sending an SMS, allowing a prompt and effective intervention.

The latter is the PC software used to connect to and control the remote devices, by means of a simple and functional interface. It is possible to customize the network of transmitters, group the stations in sub-groups, display the status and any alarm, check an event log, switch on and off the devices and change their settings (such as power).

Although made for controlling Elettronika equipment, the RCS provides enough flexibility to allow the control of third-party or general-purpose devices (such as intruder alarms, air-conditioning systems and so on).

\section{REFERENCES}

[1] Giuseppe Modugno, "RCU Telemetry Accesories," Elettronika Group, Rev. 2.1., pp. 18-48, 01/09/08.

[2] Giuseppe Modugno, "ERCoS v3.0 Elettronika Remote Control Software," Elettronika, Rev. 3.0., pp. 3-40, 08/07/08.

[3] http://www.elettronika.it/index.php?vers=Eng\&cat=4\&id_cat=1 this is the download link for the ERCoS demo software.

[4] Eric Burger, "Ubiquitous Reach and Remote Control Of Devices", VDM Verlag Dr. Mueller e.K., October 2008

\section{AUTHORS}

Alexandra Teodor is with the Elettronika Research, Brasov, Romania (a.teodor@elettronikaresearch.com).

Atilla Valadi is with the Elettronika Research, Brasov, Romania (a.valadi@elettronikaresearch.com).

Doru Ursutiu is with the University Transilvania, Dept. Physics, Brasov, Romania (udoru@unitbv.ro).

Giuseppe Modugno is with the Elettronika,Dept. Research \& Development, Palo del Colle, Italy (g.modugno@elettronika.it).

Marco Fiore is with the Elettronika, Dept. Research \& Development, Palo del Colle, Italy (m.fiore@elettronika.it).

Manuscript received 15 April 2009. Published as submitted by the authors. 